\title{
Effects of plasma kinetic modelling on performance characterization of plasma actuators for active flow control
}

\author{
Donato Fontanarosa $^{1, *}$, Giacomo $_{\text {Cinieri }}{ }^{1}$, Maria Grazia De Giorgi ${ }^{1}$ and Antonio Ficarella ${ }^{1}$ \\ ${ }^{1}$ UNIVERSITA’ DEL SALENTO, Department of Engineering for Innovation, 73100 Lecce, Italy
}

\begin{abstract}
This work focuses on the development of a multiscale computational fluid dynamics (CFD) simulation framework for the investigation of the effects of plasma kinetics on the performance of a microscale dielectric barrier discharge plasma actuator (DBD-PA). To this purpose, DBD-PA multi-scale dual-step modelling approach has been implemented, by considering plasma chemistry and flow dynamic. At first, a microscopic plasma model based on the air plasma kinetics has been defined and plasma reactions have been simulated in zero-dimensional computations in order to evaluate the charge density. At this aim computations have been performed using the toolbox ZDPlasKin, which solves plasma reactions by means of Bolsig+ solver. An alternate current (AC) electrical feeding has been assumed: in particular, the sinusoidal voltage amplitude and the frequency have been fixed at $5 \mathrm{kV}$ and $1 \mathrm{kHz}$ at atmospheric pressure and $300 \mathrm{~K}$ temperature in quiescent environment. The predictal charge density has been in a macroscopic plasma-fluid model based on Suzen Dual Potential Model (DPM), which has implemented in the computation fluid dynamic CFD code OpenFoam. Hence, as second step, 2D-CFD simulations of the electro-hydrodynamic body forces induced by the microscale DBDPA have been performed, based on the previously predicted charge densities at the operating conditions. Quiescent flow over a dielectric barrier discharge actuator has been simulated using the plasma-fluid model. The novel modelling framework has been validated with experimental data.
\end{abstract}

\section{Introduction}

Nowadays, dielectric barrier discharge plasma actuator (DBD-PA) technology has demonstrated to be interesting in active flow control systems for fluidic machinery and aeronautic foils applications, e.g. boundary layer transition and turbulence control $[1,2]$ and noises reduction $[3,4]$. In fact, its peculiar characteristics make this solution ideal for real time control systems because of frequencies, no moving parts, easy and cheap installation and low power consumption [5-8]. Furthermore, the recent advancements in micro-manufacturing has allowed for a multiple and distributed devices integration into more complex and actual configurations [9, 10], extending the application range to microfluidic devices and micropropulsion systems for satellites applications [11].

The effectiveness of the plasma-based active flow control strictly depends on the entity of the chemistry effects, which promotes the generation of excited and charged species allowing for the momentum and energy transfer to the flow [12]. The ionization process is driven by collisions between ions, electrons and neutral species [13] based on the Paschen's law, which relates the ionization degree (i.e. the ratio between the electron density and the neutral gas density) to the operating pressure and temperature. The last ones significantly affect the plasma actuator performance [14, 15], which also depend on geometric variables (e.g. exposed electrode area, dielectric thickness and electrodes gap [16-18]), dielectric (typically Kapton, Teflon, ceramic or PMMA) and electrodes materials (typically electroplated copper) [19]. In addition, the possibility of different electrical powering (e.g. direct current, high-voltage sinusoidal and nanosecond repetitively pulsed regimes) provide to DBD-PAs a high degree of flexibility in controlling the plasma discharge process [20-23]. Despite several demonstrations of DBD effectiveness in a laboratory scale [24], a full integration of such devices into aero-engine control systems is still far from being demonstrated [25]. To this last purpose, the development of reliable, accurate and flexible numerical models is of a primary importance since it would enable for DBD-PA performance prediction and the consequent reduction of the strong complexity affecting the DBD-PA design [26].

"Corresponding author: donato.fontanarosa@unisalento.it 
In this context, the present work aims to provide a novel numerical methodology for the analysis of the flow field modification induced by a DBD-PA: it relates the zero-dimensional analysis of the chemistry of the plasma discharge in the gas flow to momentum transfer and flow acceleration estimated by means of CFD computations. In particular, a lumped circuit model is used to predict the actual reduced electric field established into the ionization region. It is then used into the zero-dimensional solver ZDPlasKin to simulate the collisional process of the plasma discharge by means of the integrated solver Bolsig+. This allows for the estimation of the charge density produced by the plasma actuation, which is used to setup the boundary conditions of two-dimensional CFD computations where the incompressible Navier-Stokes equations are solved in combination with electric field computation based on Dual Potential Model (DPM) by Suzen [27]. The novelty of the methodology consists in numerically solving the plasma kinetics for charge density estimation in place of more expensive experimental campaigns. The proposed methodology has been validated on previous experiments concerning a micro DBDPA investigated at ambient pressure and temperature, and quiescent conditions [28-31], fed by a high-voltage (HV) alternating current (AC) sinusoidal signal. The validation enables for further investigations at different operating conditions, DBD-PA geometry and electrical feedings.

\section{Micro DBD plasma actuator}

The micro DBD-PA consists of two electroplated copper electrodes separated by glass-reinforced epoxy laminate (FR4) dielectric layer. Both electrodes own spanwise length of $0.06 \mathrm{~m}$, and they are patterned along the streamwise direction by a gap length of $0.25 \mathrm{~mm}$. In order to preserve the flow field modification induced by the micro DBD-PA from any external disturbance, the test cell was confined into a plexiglass box of dimension $0.24 \times 0.24 \times 0.25 \mathrm{~m}^{3}$. A sketch of the micro DBDPA configuration and the overall physical system is shown in Fig. 1, while Table 1 summarizes the geometrical characteristics of the micro DBD-PA.

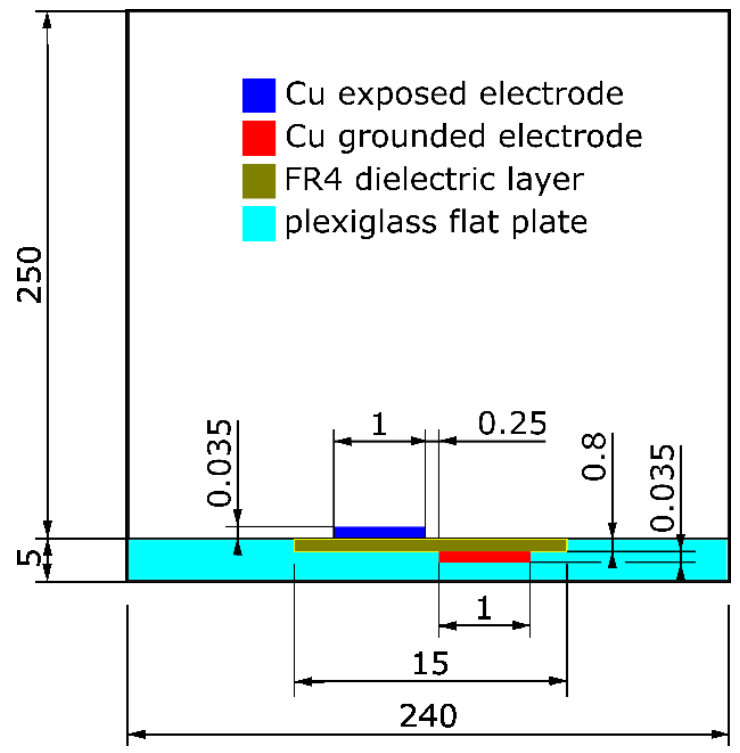

Fig.1 Sketch of the micro DBD-PA and the overall physical system. Units are in mm.

Table 1. Plasma actuator dimensions.

\begin{tabular}{|c|c|c|}
\hline Test case geometric parameters & Symbol & Length [m] \\
\hline Electrodes Spanwise length & $\mathrm{z}$ & $6 \times 10^{-2}$ \\
\hline Streamwise Electrodes gap & $\mathrm{g}_{\mathrm{el}}$ & $2.5 \times 10^{-4}$ \\
\hline Electrodes Thickness & $\mathrm{h}_{\mathrm{e}}$ & $3.5 \times 10^{-5}$ \\
\hline Dielectric thickness & $\mathrm{L}_{\mathrm{d}}$ & $8 \times 10^{-4}$ \\
\hline Electrodes length & $\mathrm{l}_{\mathrm{el}}$ & $1 \times 10^{-3}$ \\
\hline
\end{tabular}

The experimental characterization of the micro DBD-PA described above was performed on air at atmospheric pressure, temperature of $300 \mathrm{~K}$, and quiescent conditions powered by a high-voltage (HV) alternating current (AC) sinusoidal signal of amplitude from $5 \mathrm{kV}$ to $7 \mathrm{kV}$ and frequency from $1 \mathrm{kHz}$ to $2.5 \mathrm{kHz}$ [29], The induced flow was investigated by particle image velocimetry and measured velocity fields were used to estimate experimentally the time averaged induced body force 
distributions by a differential method. We refer to [29] for a more detailed description of the experimental setup and methodologies.

\section{Numerical modelling}

Plasma actuator performances heavily depend on the net charge generated during the discharge: therefore, a novel numerical methodology for the analysis of the flow field modification induced by the micro DBD-PA has been developed. It couples the zero-dimensional chemistry analysis of plasma discharge in the gas flow to CFD computations of the momentum transfer and flow acceleration induced by plasma actuation. The methodology composes of two main steps. The former aims to predict the net charge density by means of simulations of the plasma discharge process. To this purpose, zero-dimensional computations of the plasma kinetics are performed using the toolbox ZDPlasKin [32]. The solution of the lumped circuit model of the micro DBD-PA at each time step has been integrated into the main Fortran90 code of the plasma chemistry solver, in order to consider the softening effect of the plasma discharge on the reduce electric field established into the ionization region. Therefore, the plasma kinetics is solved field by ZDPlasKin at each time step drive by the predicted reduced electric field, using electron-impact reaction rates computed by Bolsig+ solver. The second numerical step consists in twodimensional CFD computations aiming to predict the impact of micro DBD plasma actuation on the fluid flow. The incompressible Navier-Stokes equations are solved in combination with electric field computation based on Dual Potential Model (DPM) by Suzen [27].

\subsection{Plasma kinetic modelling of air}

ZDPlasKin is a zero-dimensional plasma kinetic solver: it integrates the Boltzmann equation solver Bolsig+ for the electron density function in a two-temperature model, and the DVODE 90 solver, which is used for chemistry differential equation solution. The use of this toolbox assumes uniformly plasma distribution in space during its ignition as confirmed by iCCD imaging [28]. Solved Bolsig+ reactions are excitation and de excitation processes, electron-ion recombination reactions, attachment and detachment for electrons and neutral species. The resulting plasma chemistry refers to the $\mathrm{N}_{2}-\mathrm{O}_{2}$ mixture: the model consists of neutrals, metastable and charged species as detailed in Table 2 [33]. Concerning reactions, bimolecular and trimolecular nitrogen-oxygen processes, ions reactions and nitrogen oxides reactions complete the chemistry dataset whose reaction rate constants are added for each one. The electron cross-section data were retrieved from the LXCat website [34] using Phelps and Pancheshnyi databases [35].

Table 2. Reactants, elements and intermediate species (radicals, charged and excited species) involved in detailed kinetic mechanism of $\mathrm{N}_{2}-\mathrm{O}_{2}$ plasma discharge [34].

\begin{tabular}{|c|c|}
\hline Species type & Symbol \\
\hline Reactants & $\mathrm{N}_{2}, \mathrm{O}_{2}$ \\
\hline Elements & $\mathrm{O} \quad \mathrm{N} \quad \mathrm{E}$ \\
\hline Radicals & $\mathrm{O} \mathrm{NO} \mathrm{N}_{2} \mathrm{O} \mathrm{NO}_{2} \mathrm{NO}_{3} \mathrm{~N}_{2} \mathrm{O}_{5}$ \\
\hline Charged species & $\mathrm{N}^{+} \mathrm{N}_{2}^{+} \mathrm{N}_{3}^{+} \mathrm{N}_{4}^{+} \mathrm{O}^{+} \mathrm{O}_{2}^{+} \mathrm{O}_{4}^{+} \mathrm{O}^{-} \mathrm{O}_{2}^{-} \mathrm{O}_{3}^{-} \mathrm{O}_{4}^{-} \mathrm{NO}^{+} \mathrm{N}_{2} \mathrm{O}^{+} \mathrm{NO}^{-} \mathrm{N}_{2} \mathrm{O}^{-} \mathrm{NO}_{2}^{-} \mathrm{NO}_{3}^{-} \mathrm{O}_{2}^{+} \mathrm{E}$ \\
\hline Excited species & 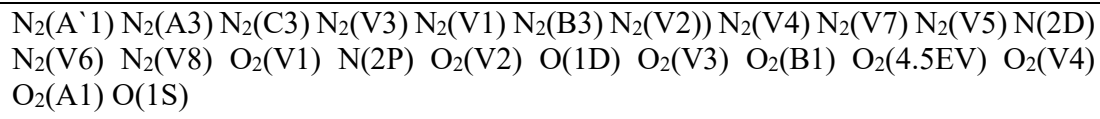 \\
\hline
\end{tabular}

Concerning initial and boundary conditions, a mixture of air $\left(\mathrm{O}_{2}+3.76 \mathrm{~N}_{2}\right)$ at pressure of $101325 \mathrm{~Pa}$ and temperature of $300 \mathrm{~K}$ were set based on experimental conditions [29], while initial electron number density was set to $10^{10} \mathrm{~m}^{-3}$ [36]. Calculations were performed with a constant time step of $10^{-7} \mathrm{~s}$. An alternate current (AC) electrical feeding has been provided with sinusoidal voltage amplitude equal to $5 \mathrm{kV}$ and frequency of $1 \mathrm{kHz}$ in accordance with experiments [37]. The gap air potential was calculated each time step as an output of the lumped electrical equivalent circuit model sketched in Figure 2. 


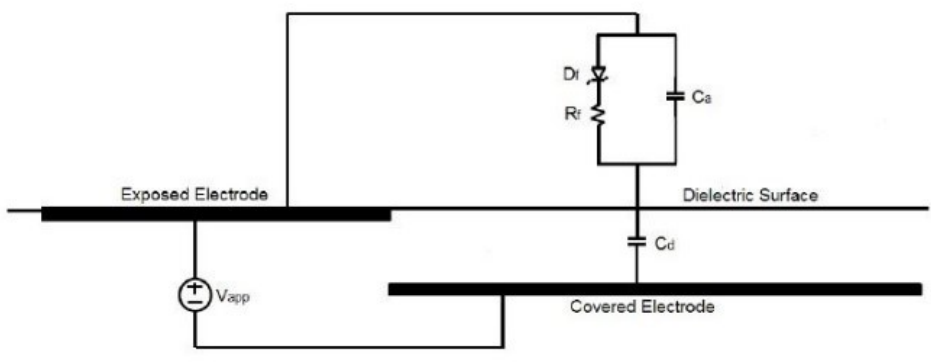

Fig.2 Equivalent lumped electrical circuit schematic of the plasma actuator [30].

It consists of a capacitor and resistor considered in parallel to describe the fluid, a capacitor for dielectric layer and a Zener diode [30]. Based on the equivalent circuit theory, when the air potential gap is greater than the $\phi_{\text {breakdown }}$ (equal to 3.0 $10^{6} \mathrm{~V} / \mathrm{m}$ [29]), the air Zener diode is activated and plasma will form [38]. However, ZDPlasKin directly solves the plasma kinetics and the breakdown phenomenon; therefore, the Zener diode behavior has been neglected, still preserving the resistive and capacitive behaviour of the micro DBD-PA. The capacitance of the dielectric surface is in series with the fluid elements. The model's resistance $R$ and capacitances of air side $C_{a}$ and dielectric side $C_{d}$, are fully estimated by the following equations [30]:

$$
\begin{gathered}
C_{\mathrm{a}}=\frac{\varepsilon_{0} \varepsilon_{\mathrm{a}} h z}{L_{\mathrm{p}}} \\
C_{\mathrm{d}}=\frac{\varepsilon_{0} \varepsilon_{\mathrm{d}} h_{\mathrm{d}} z}{L_{\mathrm{d}}} \\
R=\frac{\rho_{\mathrm{a}} L_{\mathrm{p}}}{h z}
\end{gathered}
$$

where $\varepsilon_{a}=1$ is the relative permittivity of air [27]. $\varepsilon_{0}=8.854 \times 10^{-12} \mathrm{~F} / \mathrm{m}$ is the vacuum permittivity, and $\rho_{\mathrm{a}}$ is the effective resistivity of the air was taken equal to $2.3 \cdot 10^{6} \Omega \cdot \mathrm{m}$ [29]. A sinusoidal potential is applied as follows:

$$
\phi_{\mathrm{a}}(t)=\phi_{\text {amplitude }} \sin (2 \pi \omega t)
$$

As a result, the Kirckoff's laws of the differential equation for the discharge gap potential $\Delta \phi$ is introduced at each time step for the estimation of the actual air gap potential, as follows [37]:

where:

$$
\frac{d \Delta \phi(t)}{d t}=\alpha \frac{d \phi_{a}}{d t}-\beta \Delta \phi(t)
$$

$$
\begin{gathered}
\alpha=1-\frac{C_{a}}{C_{a}+C_{d}} \\
\beta=\frac{1}{R\left(C_{a}+C_{d}\right)}
\end{gathered}
$$

Therefore, the actual reduced electric field, which drives plasma kinetics computations, is given by:

$$
E_{\mathrm{N}}=\left|\frac{\Delta \phi}{\mathrm{L}_{\mathrm{p}}} N_{\text {neutral species }}\right|
$$

where $N_{\text {neutral species }}$ is the number density of all neutral species and $L_{p}$ is the discharge gap length, estimated as the plasma height $h$ and gap electrodes $g_{e l}$.

As observed in [38] the height of the plasma value is independent of applied voltage; so it has been assumed equal to $1 x 10^{-3}$ $\mathrm{m}$ in accordance with experimental findings [30]. 
After plasma kinetics computations, the temporal evolution of all species is retrieved: this allows for the calculation of the maximum net charge density $\rho_{\mathrm{c} \text { max }}$ as follows [15]:

$$
\rho_{\mathrm{c} \max }=\max \left|q\left(\mathrm{~N}_{\text {Ions }_{+}}-\mathrm{N}_{\text {Ions_- }}-\mathrm{N}_{\mathrm{e}}\right)\right|
$$

\subsection{Implementation of plasma effects in the CFD code}

As a second step, the Suzen's Dual Potential Model (DPM) coupled with the incompressible Navier-Stokes equations in the CFD toolbox OpenFOAM v3.0.1, using a finite volume method (FVM) approach. The incompressible flow hypothesis is justified by the actual low Reynolds number $R e<200$ [40]. Heat transfer from the plasma to the neutral gas is neglected in accordance with experimental observation by [40]. The solved plasma electro-hydrodynamic (EHD) body force can be simplified as [17]:

$$
\mathbf{f}=-\rho_{\mathrm{c}} \boldsymbol{\nabla} \phi
$$

Furthermore, the Laplace equation was solved to predict the electric potential field inside the fluid domain:

$$
\boldsymbol{\nabla} \cdot\left(\varepsilon_{0} \boldsymbol{\nabla} \phi\right)=0
$$

Instead, the net charge in the fluid domain is computed by solving the Poisson's equation as follows:

$$
\nabla \cdot\left(\varepsilon_{\mathrm{r}} \nabla \rho_{\mathrm{c}}\right)=\frac{\rho_{\mathrm{c}}}{\lambda_{\mathrm{D}}}
$$

where $\varepsilon_{r}$ is the relative permittivity of the FR4 layer (set equal to 5) and $\lambda_{D}$ is the Debye's length. This last one typically ranges from $10^{-4}$ to a few $\mathrm{cm}$ [41]; based on previous sensitivity analysis [29], $\lambda_{D}$ has been set equal to $1.0 \times 10^{-3} \mathrm{~m}$. Finally, plasmainduced body force is given as follows:

$$
\mathbf{f}=-\rho_{\mathrm{c} \max } \phi_{\text {amplitude }} \rho_{\mathrm{c}} \nabla \phi \sin ^{2}(2 \pi \omega t)
$$

The plasma-induced body force is then added as external momentum source into the momentum balance equation for incompressible flow (Eq. 15). Together with the continuity equation (Eq. 14), they define the final set of governing NavierStokes equations, which are solved by means of the Pressure-Implicit with Splitting of Operators (PISO) scheme.

$$
\begin{gathered}
\boldsymbol{\nabla} \mathbf{u}=0 \\
\frac{\mathrm{d} \mathbf{u}}{\mathrm{dt}}+\boldsymbol{\nabla}(\mathbf{u} \mathbf{u})-\boldsymbol{\nabla}\left(\frac{\varrho_{\mathrm{air}}}{\mu} \nabla \mathbf{u}\right)=-\nabla p+\mathbf{f}
\end{gathered}
$$

where $\boldsymbol{u}$ is the local velocity vector in $\mathrm{m} / \mathrm{s}, \varrho_{\text {air }}$ is the air mass density expressed in $\mathrm{kg} / \mathrm{m}^{3}, \mathrm{p}$ is the local pressure in Pa, $t$ is the time in $\mathrm{s}$.

\subsection{CFD simulation setup: computational domain and boundary conditions}

Since the large span of the actuator ensures minimal three-dimensional effects [42], a two region 2D computational domain is provided. Fluid region consists of 270000 cells. Square elements with a finer resolution are close to the device upward and downstream the electrode until $14 \mathrm{~mm}$ in order to obtain the most accurate value for jet profile. Mesh was divided in more subdomains to run computations in parallel. Figure 3 shows the computational mesh with a focused view close to the micro DBD-PA. 


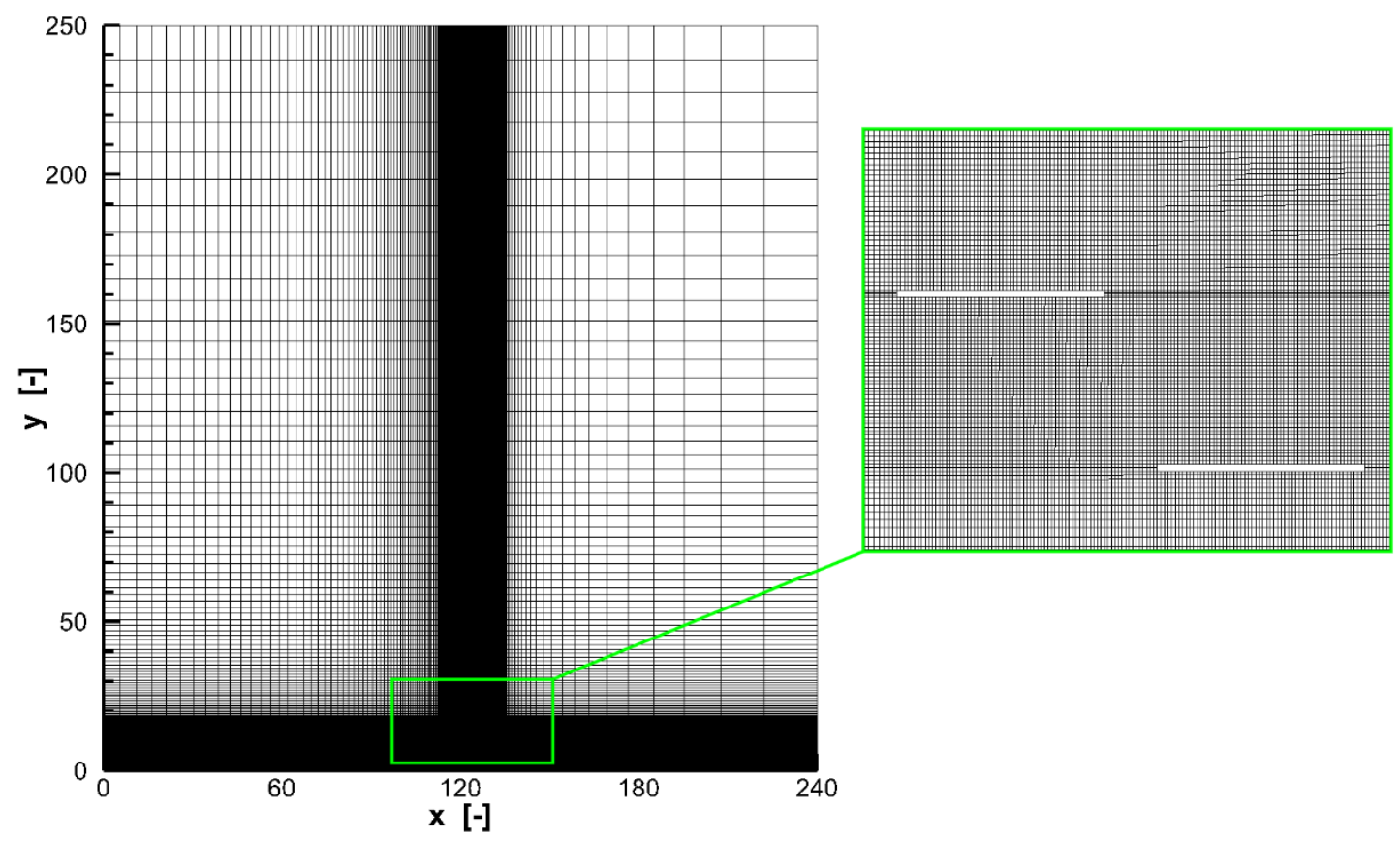

Fig.3 Computation domain and mesh.

Based on the operative conditions, the air kinematic viscosity $\mu_{\text {air }}$ and mass density $\varrho_{\text {air }}$ were assumed constant and equal to $1.58 \cdot 10^{-5} \mathrm{~m}^{2} \mathrm{~s}^{-1}$ and $1.18 \mathrm{~kg} / \mathrm{m}^{3}$, respectively.

Concerning the boundary conditions, in accordance with [21], a zero-gradient condition was imposed on all solid and fluid boundaries with the following exception:

- $\rho_{\mathrm{c}}=0 \mathrm{C} / \mathrm{m}^{3}$ on the exposed electrode surface;

- $\rho_{\mathrm{c}}=0 \mathrm{C} / \mathrm{m}^{3}$ on the dielectric surface air side, above the gap between electrodes;

- Half Gaussian spatial distribution in the downstream plasma region above the grounded electrode was set according to [27] as a half gaussian function as follows:

$$
\rho_{c}(x)=\exp \left[-\frac{\left(x-\mathrm{g}_{\mathrm{el}}\right)^{2}}{4 \sigma^{2}}\right]
$$

In Eq. 17, the standard deviation $\sigma$ describes the correct extension of the charge density [37]: it was assumed equal to $5 \cdot 10^{-4}$ according to experimental values of light emission of the our testcase [43]. The electric potential boundary conditions were set to zero-gradient on all boundaries except the electrodes: in accordance with experiments, the grounded electrode potential was fixed to $0 \mathrm{~V}$ while the amplitude of the sinusoidal high-voltage signal was imposed on the exposed electrode surface, i.e. $5000 \mathrm{~V}$. Instead, the sinusoidal high-voltage frequency was set to $1000 \mathrm{~Hz}$. No slip condition was set for the fluid to solid region boundaries and zero gradient for the other fluid borders. Initial velocity was zero because of the quiescent condition, while the pressure was set to $101325 \mathrm{~Pa}$ in the far-field of the fluid domain and wave transmissive bc was set on outer boundaries in order to not reflect waves. A variable time step was used with maximum Courant number Comax $<1.0$. It leads to a minimum time step of $2 \cdot 10^{-6}$ s. At each time step, computations were performed by ensuring residual tolerances below $10^{-6}$. The entire set of numerical schemes used in CFD computations is summarized in Table 3 .

Table 3. Numerical schemes of CFD computations.

\begin{tabular}{|c|c|}
\hline Operator & Scheme \\
\hline Temporal derivative & Euler \\
\hline Gradient & Gauss linear \\
\hline Divergence & Gauss upwind \\
\hline Laplacian & Gauss linear corrected \\
\hline Interpolation & Linear \\
\hline
\end{tabular}




\section{Results}

\subsection{Plasma modeling results}

The charge species densities was computed by equation (9). The data were smoothed with methods of noise suppression to filter instantaneous high-frequency noise outliers [44]. Best results are obtained with a 200-time steps window technique. A wider window would result in more smoothing but at the cost of more distortion of higher frequency content. The results are shown in figure 5. Starting from the charge species density, the current has been computed as [28]:

$$
i(t)=\frac{j \cdot \rho \cdot \mathrm{d} A}{N_{\mathrm{e}}}
$$

where $\mathrm{j}$ and $\mathrm{Ne}$ are computed by ZDPlasKin and shown in Figure 4, $\mathrm{dA}$ is the cross-sectional area of the plasma discharge of the elementary circuit:

$$
\mathrm{dA}=\frac{\mathrm{z} \cdot \mathrm{L}_{\mathrm{p}}}{\mathrm{n}}
$$

$\mathrm{n}$ is the number of elementary circuits composing the micro DBD-PA. Among n values in [29], we found 65 as the best agreement with experimental data. Highest charge density in each cycle $\rho_{\mathrm{c} \text { max }}$ was found to be $1 \cdot 10^{-2} \mathrm{C} / \mathrm{m}^{3}$. In figure 6 smoothed data are compared with experimental data of the current test case taken by [29]. In Figure 7 the spatial distribution of charge density and plasma force, which was computed by equation (13), are shown in the fluid area close to the electrodes. As expected, the highest force magnitude was found downstream the exposed electrode at $\mathrm{x}$ station above the grounded one $\left(\mathrm{x}=2.5 \cdot 10^{-4} \mathrm{~m}\right)$.

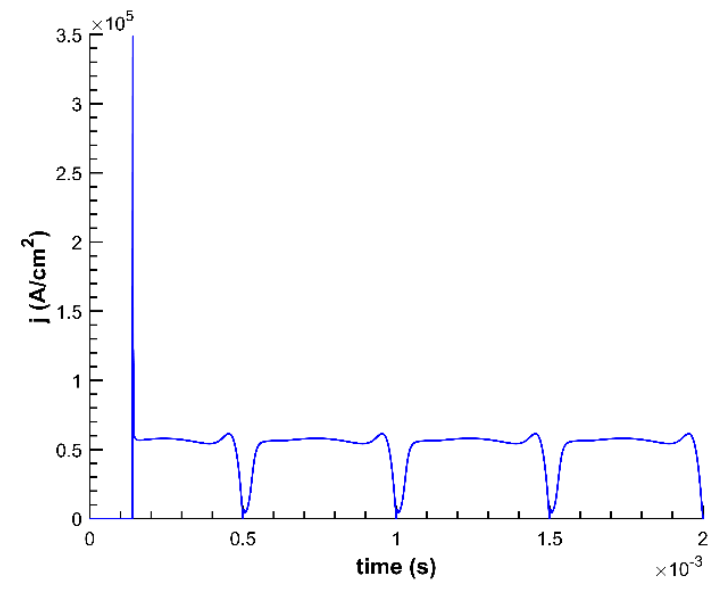

a)

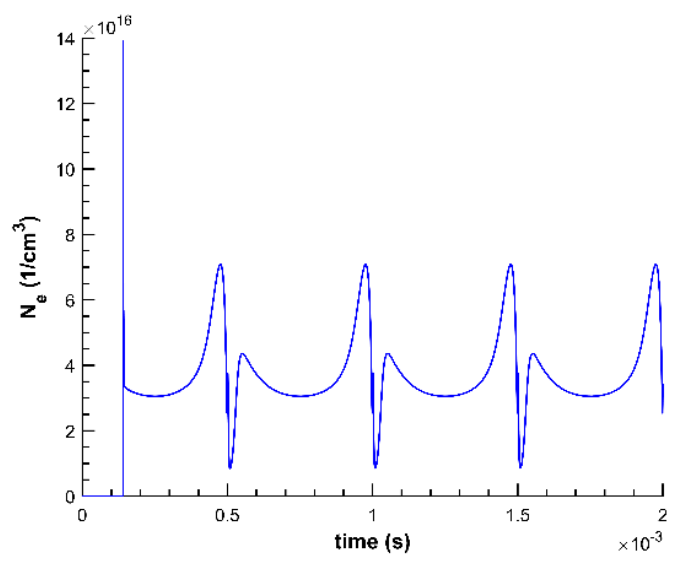

b)

Fig.4 First cycles of data computed by ZDPlasKin : a) current density; b) electrons density.

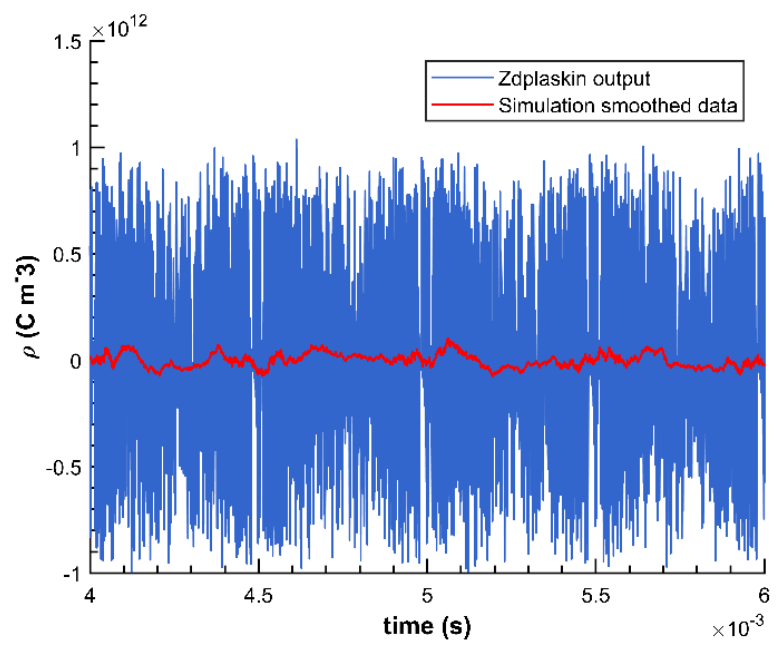

Fig.5 Predicted net charge density. 


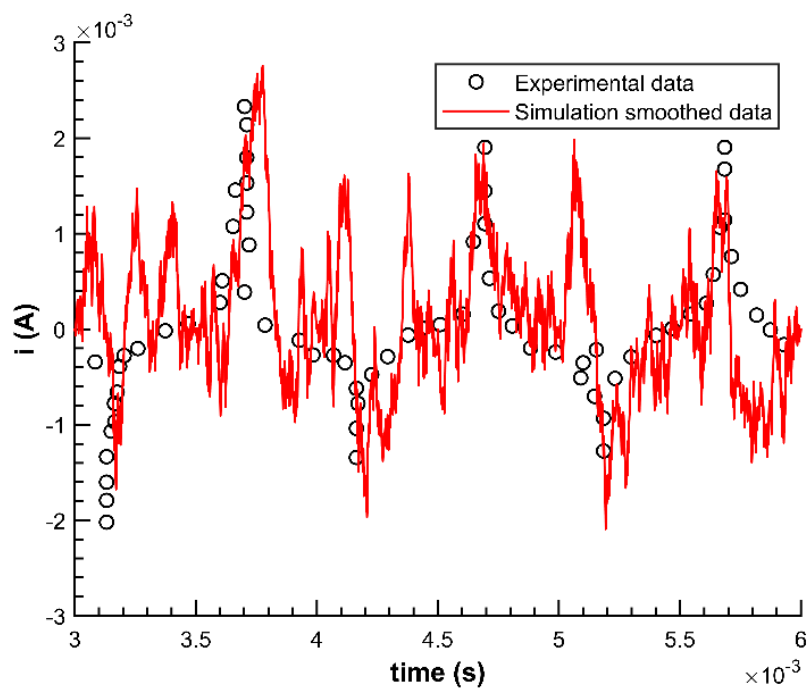

Fig.6 Experimental versus predicted current.

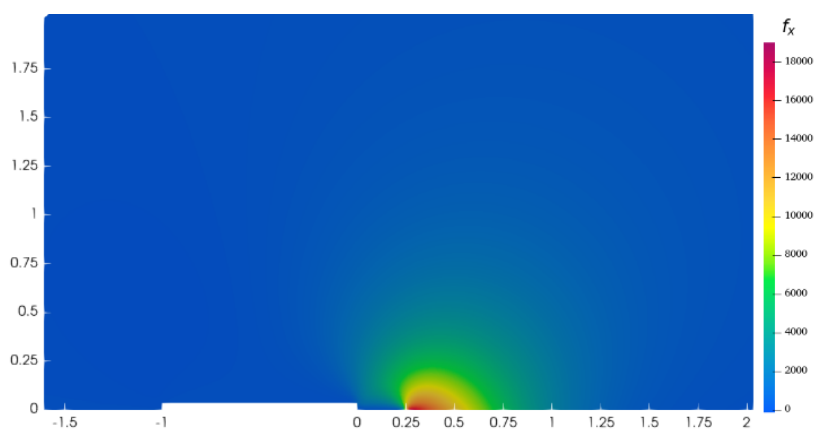

a)

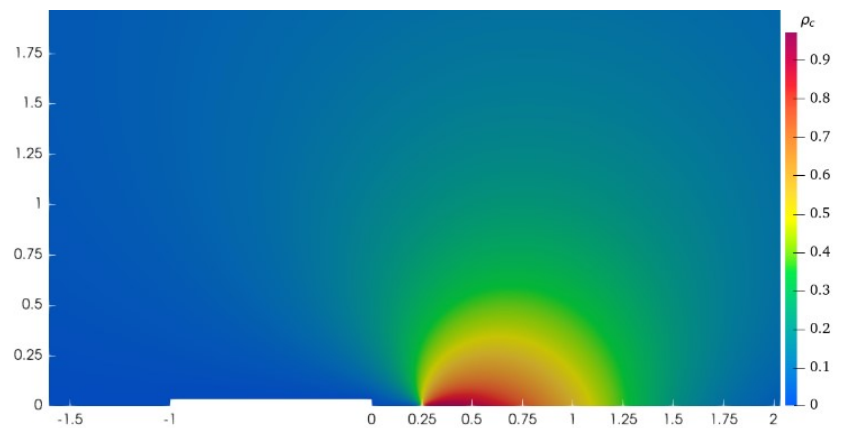

b)

Fig.7 a) Average $x$ component body force expressed in $\mathrm{N} / \mathrm{m}^{3}$. b) Average charge density spatial distribution. Coordinates are in mm.

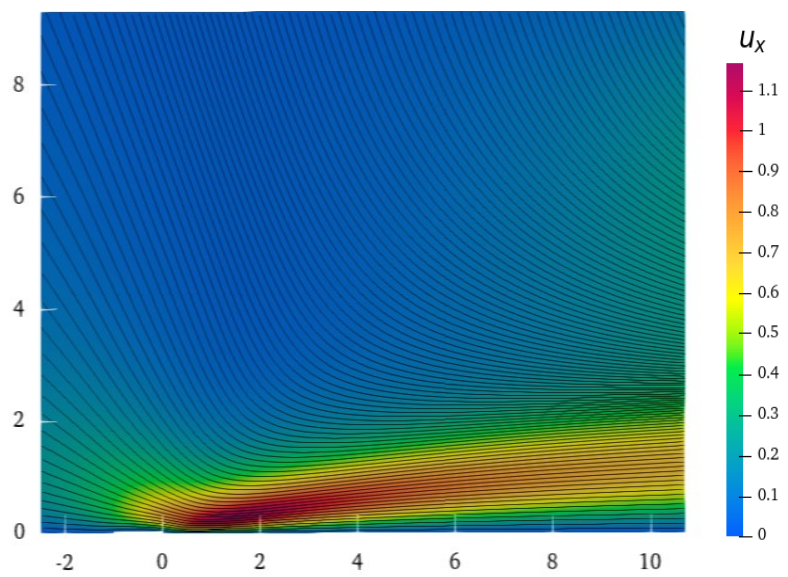

Fig.8 Streamlines in the fluid region. Coordinate are in $\mathrm{mm}$. $\mathrm{u}_{\mathrm{x}}$ is expressed in $\mathrm{m} / \mathrm{s}$.

\subsection{Flow simulations}

Computations were performed until $0.65 \mathrm{~s}$. In Fig 8 the velocity stream lines are shown. Laminar flow is proved and fluid jet extends in $\mathrm{x}$ direction and until $2 \mathrm{~mm}$ for y direction. Numerical data were compared with experimental results taken from [45] by using $3 \mathrm{~mm}$ and $7 \mathrm{~mm}$ station downstream the edge of the exposed electrode. As shown in Fig. 9 the model is able to 
match the magnitude and thickness of the wall-jet close to the wall, the velocity of the plasma jet is in a better agreement with experiments close to the wall as shown in [45].

a)

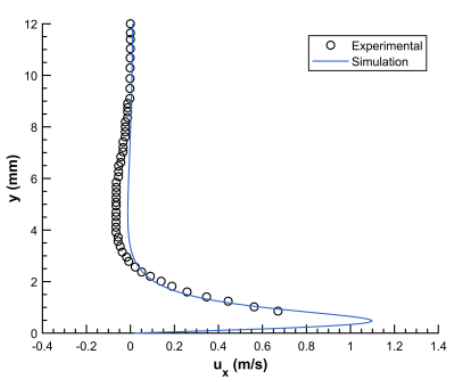

b)

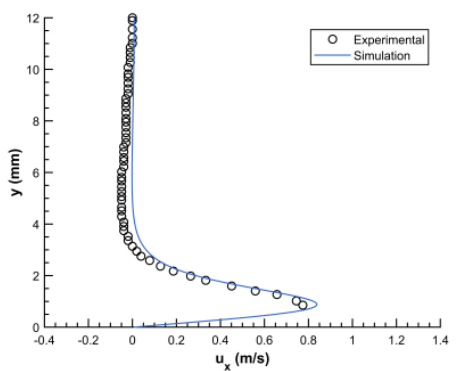

c)

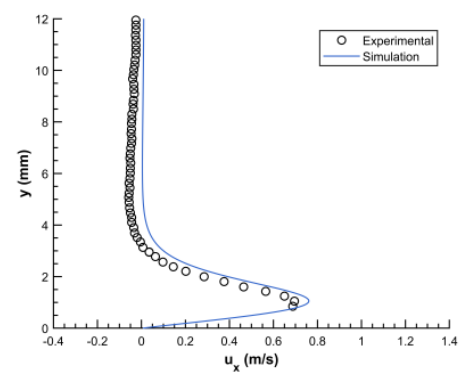

Fig.9 Plasma induced jet velocity at $0.65 \mathrm{~s}$ at three positions downstream the upper electrode: a) $\mathrm{x}=3 \mathrm{~mm} \mathrm{~b}) \mathrm{x}=7 \mathrm{~mm}, \mathrm{c}) \mathrm{x}=9 \mathrm{~mm}$.

\section{Conclusions}

The current paper aims to develop a novel multiscale modelling framework for the simulation of DBD plasma actuators, by coupling a microscopic plasma kinetic model with a macroscopic CFD plasma-fluid model, in order to have a detailed description of the plasma without computational expensive calculations.

The proposed detailed microscopic plasma kinetic model has been used to estimate the species charge density distribution profile on the dielectric surface to be used in the plasma-fluid model, based on the well-known Dual potential Model by Suzen et al. [27]. Numerical computations are validated by comparing with experimental data of the discharge current. Predictions were found to be in very good agreement thus providing an extensive validation.

Furthermore, quiescent flow over a dielectric barrier discharge actuator has been simulated using the plasma-fluid model. The streamline plots were analysed and the velocity profiles were compared with experimental data, the predictions were found to be in-line with the experiments.

\section{Notation}

$\mathrm{N}_{\mathrm{i}}$ species density

$\mathrm{T}_{\text {gas }}$ species temperature $[\mathrm{K}]$

$\mathrm{E}_{\mathrm{N}}$ reduced electric field [Td]

$\mathrm{k}$ reaction rate

e electron

$\varepsilon_{0} \quad$ vacuum permettivity $[\mathrm{F} / \mathrm{m}]$

$\mathrm{h}$ plasma height [m]

$\mathrm{d}_{\text {air }}$ density $\left[\mathrm{kg} / \mathrm{m}^{3}\right]$

$\mathrm{z}$ spanwise electrode length

$\omega$ frequency

$\mathrm{t}$ time [s]

$\mathrm{x}$ horizontal coordinate [m]

$\mathrm{y}$ vertical coordinate $[\mathrm{m}]$ i current [A]

$\mathrm{j}$ current density $\left[\mathrm{A} / \mathrm{m}^{2}\right]$

$\mathrm{n}$ circuit division parameter

$\phi_{\text {amplitude }}$ sinusoidal amplitude

$\phi_{\text {gr_el }} \quad$ grounded electrode potential

$\phi_{\text {exp_el } \quad \text { exposed electrode potential }}$

$\lambda$ debye length [m]

Re Reynolds number

f body force $\left[\mathrm{N} / \mathrm{m}^{3}\right]$

q electric elementary charge $\left[\mathrm{C} / \mathrm{m}^{3}\right]$

$\varepsilon_{\mathrm{a}}$ air dielectric coefficient

$\mathrm{p}$ pressure $[\mathrm{Pa}]$

$\mathrm{u}$ fluid velocity 


\section{References}

[1] A. Blanco, S. Roy, Numerical simulation of a free molecular electro jet, 51st AIAA Aerospace Sciences Meeting (2013).

[2] I. Moralev, V. Sherbakova, I. Selivonin, V. Bityurin, and M. Ustinov, Effect of the discharge constriction in DBD plasma actuator on the laminar boundary layer Int. J. Heat Mass Transfer. 116, pp. 1326-1340 (2018).

[3] X. Huang, X. Zhang, Streamwise and spanwise plasma actuators for flow-induced cavity noise control, Phys. Fluids 20, 037101 (2008).

[4] H.Yokoyama, I. Tanimoto, A. Iida, Experimental tests and aeroacoustic simulations of the control of cavity tone by plasma actuators. Appl. Sci., 7, 790 (2017).

[5] H. Junhui , T. C. Corke, F. O. Thomas, Actuators for Separation Control of Low-Pressure Turbine Blades, AIAA J., 44, 1, pp. 51-57 (2006).

[6] M. G. De Giorgi, S. Traficante, A. Ficarella, Performance Improvement of Turbomachinery using Plasma Actuators, GT2011-46413, Proceedings of ASME Turbo Expo 2011, pp. 369-380 (2011).

[7] M. G. De Giorgi, S. Traficante, C. De Luca, D. Bello, A. Ficarella, Active Flow Control Techniques on a Stator Compressor Cascade: a Comparison between Synthetic Jet and Plasma Actuators, GT2012-69535, Proceedings of ASME Turbo Expo 2012, pp. 439-450 (2012).

[8] F. Rodrigues, J. Páscoa J, F. Dias, Power consumption characterization of DBD plasma actuators for boundary layer control. International Conference on Engineering. 2015

[9] X. Zhang, Z. Li Z., F. Sun, Z. Zhao, C. Wang, J Hu, Numerical Investigation of Multi-SDBD Plasma Actuators for Controlling Fluctuating Wind Load on Building Roofs. Appl. Sci., 9, 3493 (2019).

[10] B. Zheng, M. Xue, G. Chang, Sliding discharge plasma actuation for forebody vortex control on a slender body at high angles of attack, AIP Adv., 10, 5, 055103 (2020).

[11] S. Jignesh, S. Roy, J. C. Zito, Design of a microNewton thrust stand for low pressure characterization of DBD actuators, in: 51st AIAA Aerospace Sciences Meeting, Grapevine, TX, AIAA Paper, 755 (2013).

[12] F. Rodrigues, J. Pascoa, and M. Trancossi, "Heat generation mechanisms of DBD plasma actuators," Exp. Therm. Fluid Sci. 90, pp. 55-65 (2018).

[13] S. Pancheshnyi, G. J. M. Hagelaar, B. Eismann, L. C. Pitchford, Computer code ZDPlasKin, University of Toulouse (2008).

[14] D. L. Tsyganov, S. Pancheshnyi, Simulation of N-atom production in dielectric-barrier discharge in nitrogen at atmospheric pressure, Plasma Sources Science and Technology, 21 (2012).

[15] M. Abdollahzadeh, J. Páscoa, P. J. Oliveira, Implementation of the Classical Plasma-Fluid Model for Simulation of the Dielectric Barrier Discharge (DBD) Actuators in OpenFOAM (2016).

[16] J. P. Murphy, J. Kriegseis P. Lavoie, Scaling of Maximum Velocity, Body Force, and Power Consumption of Dielectric Barrier Discharge Plasma Actuators via Particle Image Velocimetry, Journal of Applied Physics, 113, 24, pp. 243301-1 - 243301-10 (2013).

[17] C. L. Enloe, T. E. McLaughlin, R. D. VanDyken, K. D. Kachner, E. J. Jumper, T. C. Corke, M. Post, O. Haddad, Mechanisms and Responses of a Single Dielectric Barrier Plasma Actuator: Geometric Effects, AIAA Journal, 42, 3, pp. 595-604 (2004).

[18] F. O. Thomas, T. C. Corke, M. Iqbal, A. Kozlov, D. Schatzman, Optimization of Dielectric Barrier Discharge Plasma Actuators for Active Aerodynamic Flow Control, AIAA Journal, 47, 9, pp. 2169-2178 (2009).

[19] E. Pescini et al. Electrode material degradation monitoring for durable dielectric barrier discharge plasma actuators manufacturing. 54th AIAA Aerospace Sciences Meeting (2016).

[20] J. R. Roth, D. M. Sherman, S. P. Wilkinson, Electrohydrodynamic Flow Control with a Glow-Discharge Surface (2000).

[21] T. C. Corke, D. A. Cavalieri, E. Matlis, Boundary Layer Instability on a Sharp Cone at Mach 3.5 with Controlled Input (2001).

[22] M. L. Post, T. C. Corke, Separation Control on High Angle of Attack Airfoil Using Plasma Actuators, AIAA 20031024 (2003). 
[23] L. S. Hultgren, D. Ashpis, Demonstration of Separation Delay with Glow-Discharge Plasma Actuators (2003).

[24] G. Touchard, Plasma actuators for aeronautics applications-state of art review, IJ PEST 2 (2008).

[25] D. E. Ashpis, D. R. Thurman, Dielectric Barrier Discharge (DBD) Plasma Actuators for Flow Control in Turbine Engines: Simulation of Flight Conditions in the Laboratory by Density Matching, International Journal of Turbo \& Jet-Engines, 157-173 (2019).

[26] T. Brauner, S. Laizet, N. Benard, E. Moreau, Modelling of dielectric barrier discharge plasma actuators for direct numerical simulations. 8th AIAA Flow Control Conference, p. 3774 (2016).

[27] Y. B. Suzen, P. G. Huang, J. D. Jacob, D. Ashpis, Numerical simulations of plasma based flow control applications, in AIAA Fluid Dyn. Conf. Exhibit, Toronto (2005).

[28] G. Nerettia, M. Taglioli, C. A. Borghi, Experimental determination and numerical evaluation under simplifying assumptions of the ozone concentration in an atmospheric-pressure air DBD plasma, EPJ D (2018).

[29] M. G. De Giorgi, E. Pescini, F. Marra, A. Ficarella, Plasma actuator scaling down to improve its energy conversion efficiency for active flow control in modern turbojet engines compressors, Appl. Therm. Eng., 106 (2016).

[30] T. Underwood, S. Roy, B. Glaz, Development of a Lumped Element Circuit Model for Approximation of Nanosecond Pulsed Dielectric Barrier Discharges, in 51st AI Aerospace Sciences Meeting including the New Horizons Forum and Aerospace, Grapeville, TX (2013).

[31] M. Abdollahzadeh, J. Páscoa, P. J. Oliveira, Numerical investigation on efficiency increase in high altitude propulsion systems using plasma actuators, in: ECCOMAS European Congress on Computational Methods in Applied Sciences and Engineering, pp. 6563-6581 (2012).

[32] URL: https://www.ZDPlasKin.laplace.univ-tlse.fr/n2-o2-.

[33] URL: www.lxcat.net.

[34] M. Capitelli, C.M. Ferreira, B.F. Gordiets, A.I. Osipov, Plasma Kinetics in Atmospheric Gases, Berlin: Springer (2000).

[35] N. Minesi, P. Mariotto, G. D. Stancu, C. O. Laux, Role of the excited electronic states in the ionization of ambient air by a nanosecond discharge, AIAA Scitech 2020 Forum, (2020).

[36] D. M. Orlov, Modelling and Simulation of Single Dielectric Barrier Discharge Plasma Actuators, in Ph.D. Dissertation, University of Nothe Dame (2006).

[37] M. G. De Giorgi, E. Pescini, F. , Marra, A. Ficarella, Experimental and Numerical Analysis of a Micro Plasma Actuator for Active Flow Control in Turbomachinery, in Proceedings of the ASME Turbo Expo. 2. 10.1115/GT2014-25337 (2014).

[38] D. J. Griffiths, Introduction to Electrodynamics, Upper Saddle River, NJ: Prentice Hall (1999).

[39] D.V. Roupassov, A.A. Nikipelov, M.M. Nudnova, Starikovskii A. Yu., Flow separation control by plasma actuator with nanosecond pulse periodic discharge, Gas Discharges and Their Applications, 17th International Conference, pp.609-612, 7-12 Sept. (2008).

[40] E. Pescini, F. Marra, M. G. De Giorgi, L. Francioso, A. Ficarella, Investigation of the boundary layer characteristics for assessing the DBD plasma actuator control of the separated flow at low Reynolds numbers, Experimental Thermal and Fluid Science, 81, pp. 482-498 (2017).

[41] S. Bürkle, Environmental Impacts on Dielectric Barrier Discharge Plasma Actuators. MS thesis. Technische Universität (2013).

[42] M. Kotsonis, S. Ghaemi, L. Veldhuis, F. Scarano, Measurement of the Body Force Field of Plasma Actuators, J. Phys. D: Appl. Phys., 44 , 4, 045204 (2011).

[43] D. Orlov, T. Apker, C. He, H. Othman, T. C. Corke, Modeling and Experiment of Leading Edge Separation Control Using SDBD Plasma Actuators, AIAA 45th Aerospace Sciences Meeting Reno, Nevada (2007).

[44] D. E. Ashpis, M. C. Laun, E. L. Griebeler, Progress Toward Accurate Measurement of Dielectric Barrier Discharge Plasma Actuator Power, AIAA J. (2017).

[45] E. Pescini, D.S. Martínez, M.G. De Giorgi, A. Ficarella, Optimization of micro single dielectric barrier discharge plasma actuator models based on experimental velocity and body force fields. Acta Astronaut. 116, p. 318-332 (2015) 